\title{
Espondilite Anquilosante: Investigação Familiar de Aspectos Clínicos, Imunogenéticos e Radiológicos ${ }^{(*)}$
}

\author{
Ankylosing Spondylitis: Familial Investigation \\ of Clinical, Immunogenetic and Radiological Aspects
}

\author{
Rejane Maria Rodrigues de Abreu Vieira ${ }^{(1)}$, Jorge Elias Jr. ${ }^{(2)}$, \\ Marcello Henrique Nogueira Barbosa ${ }^{(3)}$ e Júlio César Voltarelli( ${ }^{(4)}$
}

\section{RESUMO}

Objetivo: avaliar familiares de primeiro grau de pacientes portadores de espondilite anquilosante (EA), em relação a alterações clínicas, imunogenéticas e radiológicas. Métodos: foram avaliados 14 pacientes portadores de EA, acompanhados no Hospital das Clínicas da FMRP-USP e 30 familiares de primeiro grau destes pacientes, pela história clínica e exame físico, tipificação do antígeno HLA-B27 (por microlinfocitotoxicidade e por citometria de fluxo), radiografia simples das articulações sacroilíacas e, em oito familiares, ressonância magnética (RM) da mesma região. Resultados: observou-se que 47\% (14/30) dos familiares apresentavam dor lombar com característica inflamatória crônica e 20\% deles (6/30) apresentavam evidências radiológicas de sacroiliite bilateral. $\mathrm{O}$ diagnóstico de espondilite anquilosante (EA) foi confirmado em 10\% dos familiares (3/30). O antígeno HLA-B27 estava presente em 60\% dos familiares testados pela técnica de linfocitotoxicidade ou por citometria de fluxo. A RM não se mostrou superior à radiologia convencional na detecção precoce de alterações sacrilíacas. Conclusões: a avaliação de familiares de primeiro grau de pacientes portadores de EA pode revelar formas paucissintomáticas ou subclínicas da doença em uma proporção significativa de sujeitos.

Palavras-chave: espondilite anquilosante, sacroiliite, HLA-B27, citometria de fluxo, ressonância nuclear magnética e investigação familiar.

\begin{abstract}
Objective: to evaluate first-degree relatives of ankylosing spondylitis (AS) patients regarding to clinical, immunogenetic and radiological aspects. Methods: fourteen patients with AS followed at the University Hospital of the Ribeirão Preto Medical School, University of São Paulo, Brazil (FMRP-USP) and thirty first-degree relatives were evaluated by clinical history, physical examination, HLA-B27 typing (by lymphocytotoxicity and flow cytometry), plain radiography and magnetic resonance imaging (MRI) of sacroiliacal joints. Results: we found that $47 \%(14 / 30)$ of the relatives presented low back pain with inflammatory characteristics, 20\% (6/30) fulfilled displayed radiological features of bilateral sacroiliitis, and 10\% (3/30) fulfilled the criteria for ankylosing spondylitis. Sixty percent of the relatives were HLA-B27 positive, verified either by lymphocytotoxicity or flow cytometry. MRI was not more sensitive than conventional radiography in the detection of early changes of sacroiliitis. Conclusions: the evaluation of first-degree relatives of patients with ankylosing spondylitis may reveal oligosymptomatic and subclinical forms of the disease in a significant proportion of subjects.
\end{abstract}

Keywords: ankylosing spondylitis, sacroiliitis, HLA-B27, flow cytometry, magnetic resonance imaging, familial investigation.

\footnotetext{
* Divisão de Imunologia Clínica, Departamento de Clínica Médica, Faculdade de Medicina de Ribeirão Preto da Universidade de São Paulo (FMRP-USP). Recebido em 03/02/03. Aprovado, após revisão, em 07/07/03.

1. Mestre em Clínica Médica pela Faculdade de Medicina de Ribeirão Preto, Universidade de São Paulo e Preceptora da Residência Médica em Reumatologia do Hospital Geral de Fortaleza.

2. Professor Assistente de Radiologia do Departamento de Clínica Médica da FMRP-USP.

3. Médico Radiologista do HCFMRP-USP.

4. Professor Associado da Divisão de Imunologia Clínica do Departamento de Clínica Médica da FMRP-USP.

Endereço para correspondência: Dr. Júlio C. Voltarelli. Hemocentro Regional - Campus USP, CEP 14051-140, Ribeirão Preto, SP; e-mail: jcvoltar@fmrp.usp.br
} 


\section{INTRODUÇÃO}

Considerada o protótipo das espondiloartropatias, a espondilite anquilosante (EA) foi reconhecida há milhares de anos pelos achados em estruturas esqueléticas encontradas em museus, mas somente no final do século XIX uma descrição clínica acurada da doença foi realizada ${ }^{(1)}$.

Formas paucissintomáticas e subclínicas da doença são freqüentes, gerando uma grande população de pacientes sem reconhecimento da doença, incluindo familiares de pacientes diagnosticados ${ }^{(2)}$. A história natural da doença é, na maioria das vezes, benigna, pois em menos de $20 \%$ dos pacientes ocorre progressão para uma forma incapacitante ${ }^{(3)}$. Similarmente a algumas afecções em que a etiologia não está claramente definida, o diagnóstico de EA é baseado em aspectos clínicos e radiológicos, que são considerados indispensáveis, de acordo com os critérios vigentes de Nova York ${ }^{(4,5)}$.

Ultimamente, várias técnicas de imagem têm sido empregadas para avaliar diferentes aspectos patológicos das articulações sacroilíacas e da coluna vertebral, no sentido de melhorar a sensibilidade diagnóstica para afecções dessas regiões. Desse modo, imagens de ressonância nuclear magnética (RM) são mais eficientes que as da radiologia convencional e a tomografia computadorizada na discriminação entre diferentes lesões de tecidos moles, tais como fibrose e inflamação, proporcionando diagnóstico mais precoce das sacroiliites e diferenciação entre lesões ativas e inativas, como revisado por Carvalho et al. ${ }^{(6)}$. Particularmente, a RM dinâmica ${ }^{(7)}$, com imagens rápidas, realizada com contraste paramagnético (gadolínio), aperfeiçoou o estudo da inflamação das articulações sacroilíacas, apesar de ainda ser controvertida na literatura sua utilidade na investigação clínica da sacroiliite, em virtude do alto custo e do desconforto para o paciente ${ }^{(6-10)}$.

Há fortes evidências do papel de fatores genéticos na etiopatogênese da EA, principalmente do antígeno HLAB27, que é encontrado em elevada porcentagem de pacientes com a doença ( $90 \%$ em caucasóides versus 4\% a 6\% da população geral ${ }^{(11-14)}$. No Brasil, vários trabalhos avaliaram a freqüência do antígeno HLA-B27 em pacientes portadores de EA: esta freqüência foi de 82,3\% em caucasóides estudados na cidade de São Paulo ${ }^{(15)}, 75 \%$ em pacientes predominantemente caucasóides estudados em Curitiba$\mathrm{PR}^{(16)}, 66,6 \%$ em 30 pacientes (18 brancos, 12 negros) estudados em Recife-PE ${ }^{(17)}, 65 \%$ em um estudo realizado em Belo Horizonte-MG ${ }^{(18)}$ e $78 \%$ em outro estudo realizado em Campinas-SP ${ }^{(19)}$. Neste último trabalho, $14,3 \%$ dos pacientes tinham história familiar da doença, enquanto no trabalho de Chahade et al. é apresentada, em uma família, correlação entre HLA-B27-CW2 com a EA e de HLA-B27BW21 com tuberculose familiar; finalmente, entre os 53 pacientes analisados por Houli et al. no Rio de Janeiro ${ }^{(20)}$, havia dois pares de irmãos afetados, sendo um deles de gêmeos HLA-B27 positivos, com evoluções diferentes da doença. Essas são as únicas referências que encontramos na literatura nacional e latino-americana de epidemiologia familiar na EA. Em média, 1\% a 2\% de adultos HLA-B27 positivos desenvolvem a doença na população geral e 10\% a 30\% no grupo de familiares de primeiro grau de pacientes portadores de $\mathrm{EA}^{(14)}$. A detecção do antígeno HLA-B27 é realizada classicamente pelo ensaio de microlinfocitotoxicidade dependente do complemento, descrito por Terasak ${ }^{(21)}$, mas, recentemente, outros métodos, como os citométricos (FACS), imunoenzimáticos (ELISA) e moleculares (PCR-SSP e PCR-SSO) ${ }^{(22-29)}$ têm sido empregados em rotina e investigação clínicas.

Diante da escassez de estudos familiares sobre a EA no Brasil, investigamos a frequiência da doença sintomática, paucissintomática e subclínica em familiares de primeiro grau de probandos. Avaliamos também a eficácia da RM, em relação à radiologia convencional, para detectar alterações inflamatórias precoces das articulações sacroilícas e da citometria de fluxo para detecção do antígeno HLAB27 em relação ao método convencional de microlinfocitotoxicidade.

\section{PACIENTES E MÉTODOS}

Foram estudados 44 sujeitos, compreendendo 30 familiares de primeiro grau e 14 pacientes com diagnóstico prévio de EA, segundo os critérios modificados de Nova York $^{(4,5)}$ acompanhados no Ambulatório de Doenças Reumáticas do Hospital das Clínicas da HCFMRP-USP por um período de 6 meses a 30 anos. O protocolo de estudo foi aprovado pelo Comitê de Ética em Pesquisa do HCFMRP-USP.

Foram avaliadas apenas as famílias em que foi possível estudar todos os familiares de primeiro grau, com exceção de pai e mãe, sendo, por isso, excluídas as famílias muito numerosas, com mais de 10 indivíduos. Oitenta e seis por cento $(12 / 14)$ dos pacientes e $87 \%$ dos familiares $(26 / 30)$ estudados eram do sexo masculino. Setenta e nove por cento dos pacientes eram brancos e $21 \%$ mulatos, enquanto 
entre os familiares de primeiro grau $67 \%$ eram brancos e $33 \%$ eram mulatos, sendo o grau de parentesco o seguinte: 16 irmãos, 3 irmãs, 9 filhos, 1 filha e 1 pai.

Os familiares foram chamados ao HCFMRP e submetidos aos mesmos procedimentos dos pacientes (história clínica, exame físico, exames laboratoriais e radiológicos). Os critérios para caracterização da lombalgia inflamatória crônica (LIC) foram: dor ou desconforto lombar, de início antes dos 40 anos e início insidioso; duração mínima de 3 meses; rigidez matinal lombar e melhora com o exercício. No exame físico foi feita avaliação da coluna cervical, torácica e lombar (lordose, distância dedo-chão, teste de Schober) e das articulações sacroilíacas (sinais de Menell e de Gaenslen e teste de Patrick).

Todos os pacientes foram submetidos à medida da velocidade de hemossedimentação (método de Wintrobe) e da detecção do fator reumatóide (teste do látex), além da pesquisa do antígeno HLA-B27 pelo método clássico de microlinfocitotoxicidade ${ }^{(21)}$ e por citometria de fluxo. Neste último, foram utilizados os anticorpos monoclonais antiHLA-B27 ligado à fluoresceína e o anti-CD3 associado à ficoeritrina e a análise foi feita no citômetro de fluxo FACScan (Becton-Dickinson) utilizando o software HLA-B27 do fabricante (HLA-B27 kit for in vitro diagnostic use, Becton Dickinson).

O exame radiológico convencional das articulações sacroilíacas, por meio da incidência de Ferguson, foi realizado em todos os participantes e analisados por dois médicos radiologistas, utilizando os critérios de Nova York, classificados em graus ( $0 \mathrm{a}$ IV). Os exames foram analisados sem o conhecimento da identidade ou do quadro clínico do sujeito do estudo (paciente ou familiar) e, em caso de discordância de leitura, obtinha-se um consenso entre os examinadores. Um estudo das articulações sacroilíacas por RM foi realizado em oito familiares incluídos na investigação, utilizando um aparelho supercondutor de 1,5 Tesla, da marca Siemens (Enlargen, Alemanha), modelo Magneton Vision. O protocolo de RM foi baseado em sequiências pesadas em T1 e T2 e STIR (short time of inversion recovery), além da seqüência pesada em T1 com supressão de gordura, após a injeção endovenosa de contraste paramagnético (gadolínio).

\section{RESULTADOS}

Em nosso estudo, 100\% dos pacientes e 47\% (14/30) dos familiares de primeiro grau apresentavam dor lombar do tipo inflamatória com quatro ou cinco das características descritas por Calin et al. ${ }^{(30)}$. A idade média de início dos sintomas nos pacientes foi de 23 anos, enquanto nos familiares sintomáticos a média de início da lombalgia inflamatória crônica foi de 29 anos. Artrite periférica em joelhos, tornozelos ou quadris estava presente em $64 \%$ dos pacientes $(9 / 14)$ e $13 \%$ dos familiares $(4 / 30)$ e história prévia de uveíte, em $21 \%$ dos pacientes (3/14) e em 3\% (1/30) dos familiares. No exame físico, havia evidências de sacroiliite em $100 \%$ dos pacientes e em $23 \%$ dos familiares (7/30), enquanto diminuição da mobilidade de algum segmento da coluna vertebral estava presente em $71 \%$ dos pacientes $(10 / 14)$ e em apenas $12 \%(4 / 30)$ dos familiares (Tabela 1$)$. Dos 44 sujeitos estudados, apenas seis eram do sexo feminino, sendo duas pacientes e quatro familiares, duas assintomáticas e duas com lombalgia inflamatória crônica e sinais físicos de sacroiliite. Apenas um paciente apresentava fator reumatóide positivo, o que não ocorreu em nenhum dos familiares e a velocidade de hemossedimentação estava aumentada (> $30 \mathrm{~mm}$ na $1 .{ }^{\mathrm{a}}$ hora) em $11 / 14$ pacientes e em 8/30 familiares, todos sintomáticos.

Todos os pacientes e $60 \%$ dos familiares (18/30) apresentaram o antígeno HLA-B27, tanto pelos métodos de linfocitotoxicidade como pela citometria de fluxo, que mostraram uma concordância de 100\%. O marcador genético estava presente em $79 \%(11 / 14)$ dos familiares com dor lombar de padrão inflamatório, em 75\% (3/4) dos acometidos por artrite periférica e no único paciente com história prévia de uveíte.

TABela 1

CARACTERÍSTICAS CLÍNICAS E LABORATORIAIS DE PACIENTES PORTADORES DE ESPONDILITE ANQUILOSANTE E SEUS FAMILIARES DE PRIMEIRO GRAU

\begin{tabular}{lcc}
\hline & Pacientes & Familiares \\
\hline Número & 14 & 30 \\
Lombalgia inflamatória & $14(100 \%)$ & $14(47 \%)$ \\
Idade de início dos sintomas & 23 anos & 29 anos \\
Artrite periférica & $9(64 \%)$ & $4(13 \%)$ \\
Sinais físicos de sacroiliite & $14(100 \%)$ & $7(23 \%)$ \\
Sinais radiológicos de sacroiliite & $14(100 \%)$ & $6(20 \%)$ \\
$\Downarrow$ mobilidade da coluna vertebral & $10(71 \%)$ & $4(13 \%)$ \\
Critérios de NovaYork para EA & $14(100 \%)$ & $3(10 \%)$ \\
VHS $>30$ mm na 1. ${ }^{\text {a hora }}$ & $11(79 \%)$ & $8(27 \%)$ \\
\hline Antígeno HLA-B27 & $14(100 \%)$ & $18(60 \%)$ \\
\hline
\end{tabular}


Todos os pacientes portadores de EA e 20\% (6/30) dos familiares apresentavam sacroiliite (definida ou suspeita) à radiologia convencional. Entre esses, três pacientes com lombalgia inflamatória típica apresentaram sacroiliite bilateral grau II $(n=2)$ ou III $(n=1)$ e, portanto, foram diagnosticados como EA pelos critérios modificados de Nova York, compreendendo 10\% do total de familiares e $21 \%$ dos familiares sintomáticos (vide exemplo na Figura 1). Outros dois familiares apresentaram sacroiliite de grau I, sendo um deles sintomático e um de grau II, este assintomático. Ressonância magnética foi realizada em oito familiares, incluindo os três diagnosticados como EA, confirmando os achados da radiologia convencional (Figura 2). Em nenhum dos cinco familiares com radiografia normal foi evidenciada qualquer alteração nas articulações sacroilíacas pela RM.
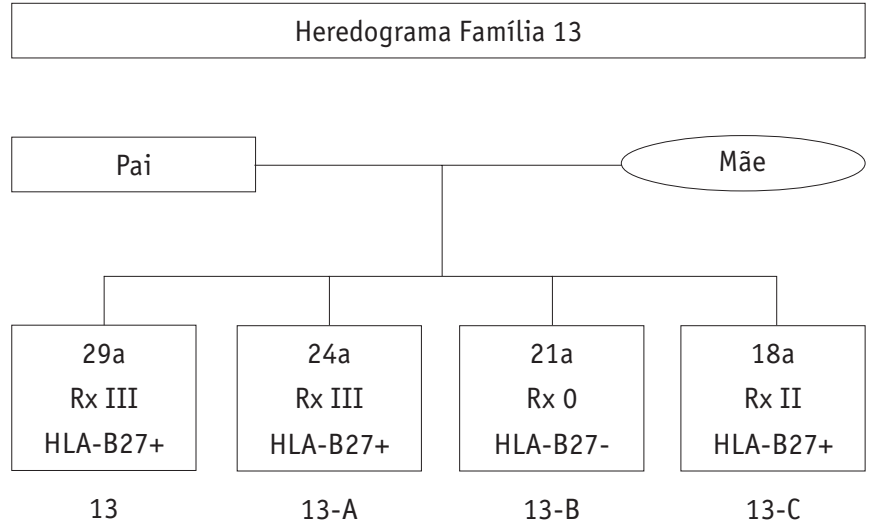

Figura 1 - Heredograma de uma família incluída no estudo, com um probando (n. ${ }^{\circ} 13,29$ anos, masculino, portador de espondilite anquilosante há 9 anos, com doença em atividade, em uso de drogas imunossupressoras) e dois irmãos sintomáticos, com alterações radiológicas e à RM de sacroiliite, mas que não haviam sido previamente diagnosticados como portadores de EA.
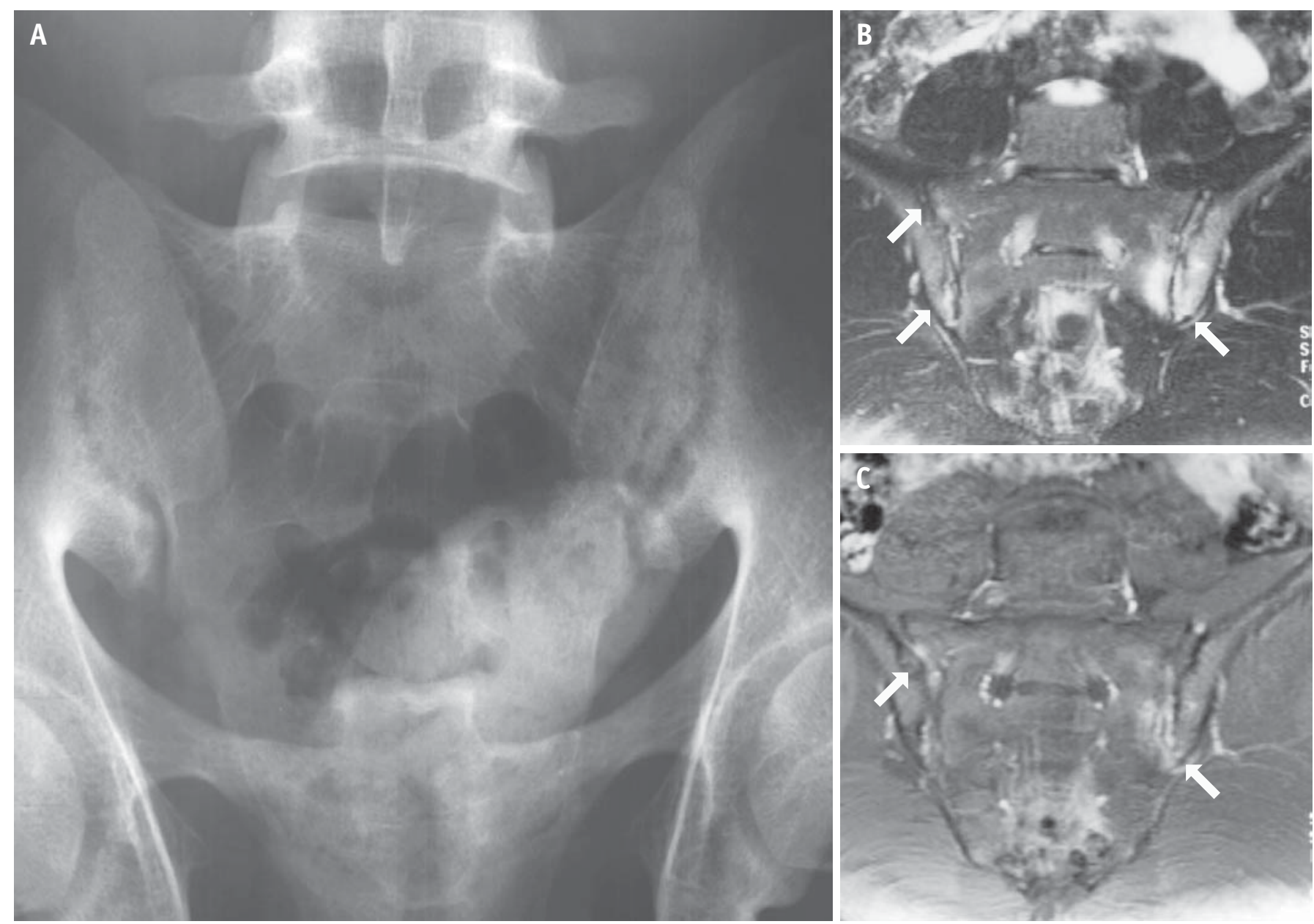

Figura 2 - Sacroiliite bilateral. (A) Radiografia simples mostrando irregularidade de contornos da superfície articular sacro-ilíaca, bilateralmente. A RM evidencia edema subcondral na seqüência pesada em T2 (B) e impregnação do contraste paramagnético na sequência pesada em T1 (C), como mostrado pelas setas. 


\section{DISCUSSÃO}

Este trabalho objetivou, primariamente, a caracterização clínica, imunogenética e radiológica de familiares de primeiro grau de pacientes com diagnóstico estabelecido de EA. $\mathrm{Na}$ avaliação inicial desses familiares, mostramos que cerca da metade deles (47\%) apresentava dor lombar com características inflamatórias crônicas, definidas por Calin et al. ${ }^{(30)}$. A literatura mostra que este sintoma constitui a forma de apresentação de $75 \%$ dos casos de EA e que a prevalência da doença é de aproximadamente $50 \%$ em indivíduos com dor lombar baixa, do tipo inflamatório e com uma história familiar positiva ${ }^{(31)}$. Em nosso estudo, 21\% dos familiares sintomáticos (3/14) preencheram os critérios modificados de Nova York para o diagnóstico de EA, enquanto um outro familiar apresentava sacroiliite suspeita (grau I). Prevê-se, portanto, que, com maior tempo de observação, este último paciente e outros desse grupo deverão apresentar alterações radiológicas diagnósticas de EA.

A literatura mostra que $10 \%$ a $20 \%$ dos casos de EA apresentam artrite periférica como manifestação inicial da doença e 35\% a desenvolvem em algum momento da evolução da doença $a^{(1,31)}$. Artrite periférica foi documentada em $64 \%$ dos nossos pacientes com EA e em quatro familiares (13\%), incluindo dois com diagnóstico definido da doença e dois com lombalgia inflamatória, porém sem critérios radiológicos da doença.

A prevalência de uveíte anterior em pacientes com EA se situa em torno de $20 \%$ a $30 \%{ }^{(32)}$. Detectamos, em nossa avaliação, 21\% de pacientes em seguimento oftalmológico por uveíte, e apenas um dos familiares de primeiro grau (3\%) tinha história de uveíte, apresentando o antígeno HLAB27, lombalgia inflamatória e sinais físicos de sacroiliite, mas sem alterações radiológicas.

Com relação ao estudo imunogenético, observamos que $100 \%$ dos pacientes avaliados, com diagnóstico definido de EA, apresentavam o antígeno HLA-B27 positivo. Na literatura, esta freqüência varia com a etnia da população estudada, situando-se em torno de $90 \%$ para a caucasóide e diminuindo muito nas populações de origem africana e oriental $^{(10-14)}$. Os estudos na população brasileira relatam uma freqüência entre $65 \%$ e $82 \%$ nas diversas regiões em que foram avaliadas ${ }^{(15-19)}$. Acreditamos que a alta freqüência de positividade do antígeno HLA-B27 em nossos pacientes seja decorrente do acaso, favorecido pelo pequeno tamanho da amostra e pelo fato de somente três pacientes não serem completamente caucasóides. Por outro lado, observamos que $60 \%$ dos familiares de primeiro grau dos probandos apresentavam HLA-B27 positivo, o que se aproxima da proporção esperada de $50 \%$. Do mesmo modo, $17 \%$ desses familiares tiveram confirmado o diagnóstico de EA, o que condiz com o achado da literatura de $10 \%$ a $30 \%{ }^{(14,33)}$. De fato, Moller et al. ${ }^{(34)}$, encontraram 20\% de EA entre os familiares de primeiro grau de pacientes, associada ao HLA-B27, considerando, assim, que a tipificação deste antígeno é provavelmente o melhor teste preditivo para a aquisição da doença, e sugerem que exames radiológicos repetidos de familiares de primeiro grau HLA-B27 negativos sejam evitados.

Em relação ao método de tipificação do HLA-B27, obtivemos uma concordância de $100 \%$ entre a técnica clássica de microlinfocitotoxicidade e a da citometria de fluxo, analisando amostras de 44 participantes do estudo, entre probandos e familiares de primeiro grau. Alguns trabalhos evidenciam uma superioridade dos métodos recentes (citometria de fluxo e PCR) em detectar o antígeno HLA-B27 em casos previamente negativos pela microlinfocitoxicidade ${ }^{(25,26)}$. Outros trabalhos, como o nosso, encontraram uma concordância total entre as técnicas de linfocitoxicidade e da citometria de fluxo, demonstrando a alta sensibilidade e a especificidade deste último método na detecção do HLA-B27 $7^{(24)}$. Assim, as vantagens práticas da citometria de fluxo, como a rapidez e a automatização da leitura, tornam-na um método bastante apropriado para a rotina clínica, o que, somadas aos resultados deste trabalho, guiaram a implantação da citometria de fluxo como método de rotina na tipificação do antígeno HLA-B27 no Laboratório de Imunogenética do HCFMRP-USP.

Em relação aos métodos de imagem, em um estudo de Bremner et al. ${ }^{(35)}$ foram encontradas 16\% de alterações radiológicas bilaterais em articulações sacroilíacas, entre 250 familiares de primeiro grau de pacientes com EA avaliados. Do mesmo modo, Moller et al. ${ }^{(34)}$ diagnosticaram $10 \%$ a $20 \%$ de EA em familiares de primeiro grau de pacientes com a doença, dependendo da presença do HLAB27. Esses achados são concordantes com os encontrados em nosso trabalho, de $20 \%$ de sacroiliite radiológica (definida ou suspeita) e de 10\% de diagnóstico de EA entre os familiares. Em nossos estudos de RM realizados em oito familiares de primeiro grau, houve uma concordância de $100 \%$ em relação à radiografia convencional, tendo-se detectado anormalidades nos três familiares diagnosticados como EA, coincidindo com as observações de Ahlstrom et al. ${ }^{(9)}$. Estes autores, em um estudo realizado 
em 27 pacientes com sintomas de sacroiliite, não encontraram diferenças significativas na sensibilidade da RNM em relação à radiografia convencional. Por outro lado, Braun et al. ${ }^{(7)}$, em um estudo posterior, utilizando imagens de RM dinâmica, com cortes rápidos, concluíram que o método era mais sensível do que a radiologia convencional para detectar precocemente a sacroiliite e poderia ser utilizado também para quantificar o processo inflamatório e monitorizar a terapêutica. Esta conclusão está anotada em recente revisão na literatura nacional, por Carvalho et al. ${ }^{(6)}$, que destacaram o valor crescente da RM, principalmente com avaliação progressiva da fase pós-contraste, quando comparada com outros métodos para detectar alterações iniciais em articulações sacroilíacas. A nossa amostragem foi bastante reduzida, de oito sujeitos, e somente foi realizado um estudo simples na fase pós-contraste, explicando o resultado encontrado. Entretanto, o uso de técnicas mais avançadas de RM para avaliação das sacroiliites e diagnóstico precoce da EA esbarra no alto custo do exame e na pouca disponibilidade do método.

\section{REFERÊNCIAS}

1. Klippel J, Dieppe PA, editors. Rheumatology. 2nd ed. St. Louis: Mosby; 1997.

2. Calin A. Seronegative spondylarthritides. Med Clin North Am 1986;70:323-36.

3. Little $\mathrm{H}$. The Natural history of ankylosing spondylitis. J Rheumatol 1988;15:1179-80.

4. Calin A. Diagnostic criteria (new and old) for the spondyloarthropathies [Editorial]. Clin Exp Rheum 1987;5:101-2.

5. Vand der Linden S, Valkenburg HA, Cats A. Evaluation of diagnostic criteria for ankylosing spondylitis: A proposal for modification of the New York criteria. Arthritis Rheum 1984;27:361-8.

6. Carvalho ES, Fernandes ARC, Ciccionele RM, Vilela SA. A sacroiliite: avaliação através dos métodos de diagnóstico por imagens de tomografia computadorizada, ressonância magnética e cintilografia óssea. Rev Bras Reumat 2001;41:109-15.

7. Braun J, Bollow M, Eggens U, et al. Use of dynamic magnetic resonance imaging with fast imaging in the detection of early and advanced sacroiliitis in spondyloarthropathy patients. Arthritis Rheum 1994;37:1039-45.

8. Docherty P, Mitchell MJ, Macmillan L, et al. Magnetic resonance imaging of sacroiliitis. J Rheumatol 1992;19:393-94.

9. Ahlstrom H, Feltelius M, Nyman R, et al. Magnetic resonance imaging of sacroiliac joint inflammation. Arthritis Rheum 1990;33:1763-69.

10. Murphey MD, Wetzel LH, Bramble JM, et al. Sacroiliitis: MR imaging findings. Radiology 1991;180:239-44.

11. Calin A. Spondyloarthropathy in caucasians and non-caucasians. J Rheumatol 1983;10:16-9.
Em conclusão, aproximadamente metade dos familiares de primeiro grau de pacientes portadores de EA apresenta sintomas sugestivos da doença, que pôde ser confirmada em $10 \%$ deles e, provavelmente, se desenvolverá em muitos outros ao longo do tempo. Esses achados reforçam o espectro de apresentações oligossintomáticas ou assintomáticas da EA e a necessidade de uma supervisão cuidadosa da família dos espondilíticos. Na nossa experiência, a citometria de fluxo foi comparável ao método clássico de detecção do antígeno HLA-B27 e a RNM não foi mais sensível do que a radiologia convencional para o diagnóstico de sacroiliite.

\section{AGRADECIMENTOS}

Às sras. Cássia M. Paula Santos, Patrícia V. B. Palma e demais funcionários dos Laboratórios de Imunogenética e de Citometria de Fluxo do Hemocentro Regional de Ribeirão Preto pela tipificação do antígeno HLA B-27. Às FUNDHERP, FAEPA-HCFMRP, FAPESP e ao CNPq pelo apoio financeiro.

12. Khan MA. Pathogenesis of ankylosing spondylitis. J Rheumatol 1993;20:1273-7.

13. Khan MA. HLA-B27 and its subtypes in world populations. Curr Opin Rheumatol 1995;7:263-9.

14. Van der Linden S, Valkenburg HA, De Jongh BM, et al. The risk of developing ankylosing spondylitis in HLA-B27 positive individuals: A comparison of relatives of spondylitis patients with the general population. Arthritis Rheum 1984;27:241-9.

15. Chahade WB, El-Khoury AB, Szwarc IS. Incidência do HLA-B27 em espondilite anquilosante em caucasóides brasileiros. Associação dos locos A, B e C/espondilite anquilosante/tuberculose pulmonar. Rev Bras Reumat 1979;19:23-6.

16. Rachid A. Espondilites anquilosantes B27 positivas e B27 negativas. Cotejo clínico, laboratorial e radiológico. Rev Bras Reumat 1979;19:107-27.

17. Ramalho ES, Freitas GG, Kosminsky S, et al. Espondilite anquilosante: estudo epidemiológico em trinta casos. Rev Bras Reumat 1989;28:1-2.

18. Carvalho MAP. Espondiloartropatias. In: Moreira C, Carvalho MAP, editores. Noções Práticas de Reumatologia. Belo Horizonte: Health; 1996. pp. 437-50. v. II.

19. Sampaio-Barros PD, Bertolo MB, Kraemer MHS. Primary Ankylosing Spondylitis: Patterns of Disease in a Brazilian Population of 147 Patients. J Rheumatol 2001;28:560-5.

20. Houli J, Klein B, Sá CAM, et al. Espondilite anquilosante: Revisão de 53 pacientes em duas diferentes épocas. Rev Bras Reumat 1980;20:75-80

21. Terasaki P. Histocompatibility Testing. Los Angeles: UCLA Tissue Typing Laboratory Publishing; 1980. 
22. Nasution AR, Marjudai A, Kunmartini S, et al. HLA-B27 subtypes positively and negatively associated with spondyloarthropathy. J Rheumatol 1997;24:1111-4.

23. Olerup O. HLA-B27 typing by a group-specific PCR amplification. Tissue Antigens 1994;43:253-6.

24. Albrecht J, Müller H. HLA-B27 typing by use of flow cytofluorometry. Clin Chem 1987;33:1619-1923.

25. Collantes-Estévez E, Gonzalez-Fernandez R, Muñoz-Gomariz E, et al. Differences in lymphocyte typing for the antigen HLA-B27 resulting from the particular technique used in patients with ankylosing spondylitis. British J Rheumatol 1997;36:1336-8.

26. Kirveskari J, Kellner H, Wuorela M, et al. False-negative serological HLA-B27 typing results may be due to altered antigenic epitopes and can be detected by polymerase chain reaction. British $\mathrm{J}$ Rheumatol 1997;36:185-9.

27. Chou CT, Tsai YF, Liu J, et al. The detection of HLA-B27 antigen by immunomagnetic separation and enzyme-linked immunosorbent assay - comparison with a flow cytometry procedure. J Immunol Methods 2001;255:15-22.

28. Sporleder H, Castro S, Rodrigues $H$, et al. Determinação de antígenos HLA-B27 por citometria de fluxo: um teste seguro? I Congresso Ibero-Latino-Americano de Citometria de Fluxo; 1997; Guarujá, São Paulo, Brasil. p. 40.

29. Su-Jen C, Ning-Sheng L, Jen-Pey S, et al. Two color analysis of HLA-B27 antigen by flow cytometer-A comparative study by conventional microlymphocytoxicity, DNA genotyping polimerase chain reaction and flow cytometric measurement. J Clin Lab Analysis 1997;11:369-73.
30. Calin A, Porta MSJ, Frie JF, et al. Clinical history as a screening test for ankylosing spondylitis. JAMA 1977;13:2613-4.

31. Van der Linden S. Ankylosing spondylitis. In: Kelley WN, et al, editors. Textbook of rheumatology. 5th ed. Philadelphia: WB Saunders CO; 1996.

32. Rosenbaum JT. Characterization of uveitis and spondyloarthritis. J Rheumatol 1998;16:792-6.

33. Braun J, Bollow M, Remlinger G, et al. Prevalence of spondyloarthropathies in HLA-B27 positive and negative blood donors. Arthritis Rheum 1998;41:58-67.

34. Moller P, Vinge O, Dale K, et al. Family studies in Bechterew's Syndrome (ankylosing spondylitis). I- Prevalence of symptoms and signs in relatives of HLA-B27 positive probands. Scand J R heumatol 1984;13:1-10.

35. Bremner JM, Emery AEH, Kellgren JH, et al. In: Bennett PH, Wood PHN, editors. Population studies of the rheumatic diseases. Amsterdam; 1968. p.299.

36. Moller P, Vinge O, Dale K, et al. Family studies in Bechterew's Syndrome (ankylosing spondylitis). II- Prevalence of symptoms and signs in relatives of HLA-B27 negative probands. Scand J Rheumatol 1984;13:11-4. 